\title{
Major expansion for Yorkshire dental group
}

ASR Dental, a dental group with practices across Yorkshire, is building a new private clinic in Huddersfield, and has added two Yorkshire Smiles surgeries in Leeds and Barnsley to its portfolio. The expansion drive is expected to create more jobs and boost the group's turnover by $25 \%$.

The firm's new clinic on Thornhill Road in Huddersfield, will serve as a specialist provider of restorative and cosmetic treatments as well as dental implants. It will feature six surgery rooms and will accept referrals from its group of practices.

Meanwhile, the company has acquired NHS dental group Yorkshire Smiles, which has two practices, in Leeds and Barnsley. The Barnsley site will be its first clinic in South Yorkshire.

The expansion plans were supported by FTA Law, led by Thomas Coates (Corporate) and Carley Jackson (Commercial Property) and supported by Lloyds Bank Commercial Banking, with a multi-million-pound loan.

Dr Altaf Hussain, director at ASR Dental, said: 'Our latest growth drive, including our first new-build surgery, is a huge milestone for the group. We've steadily acquired

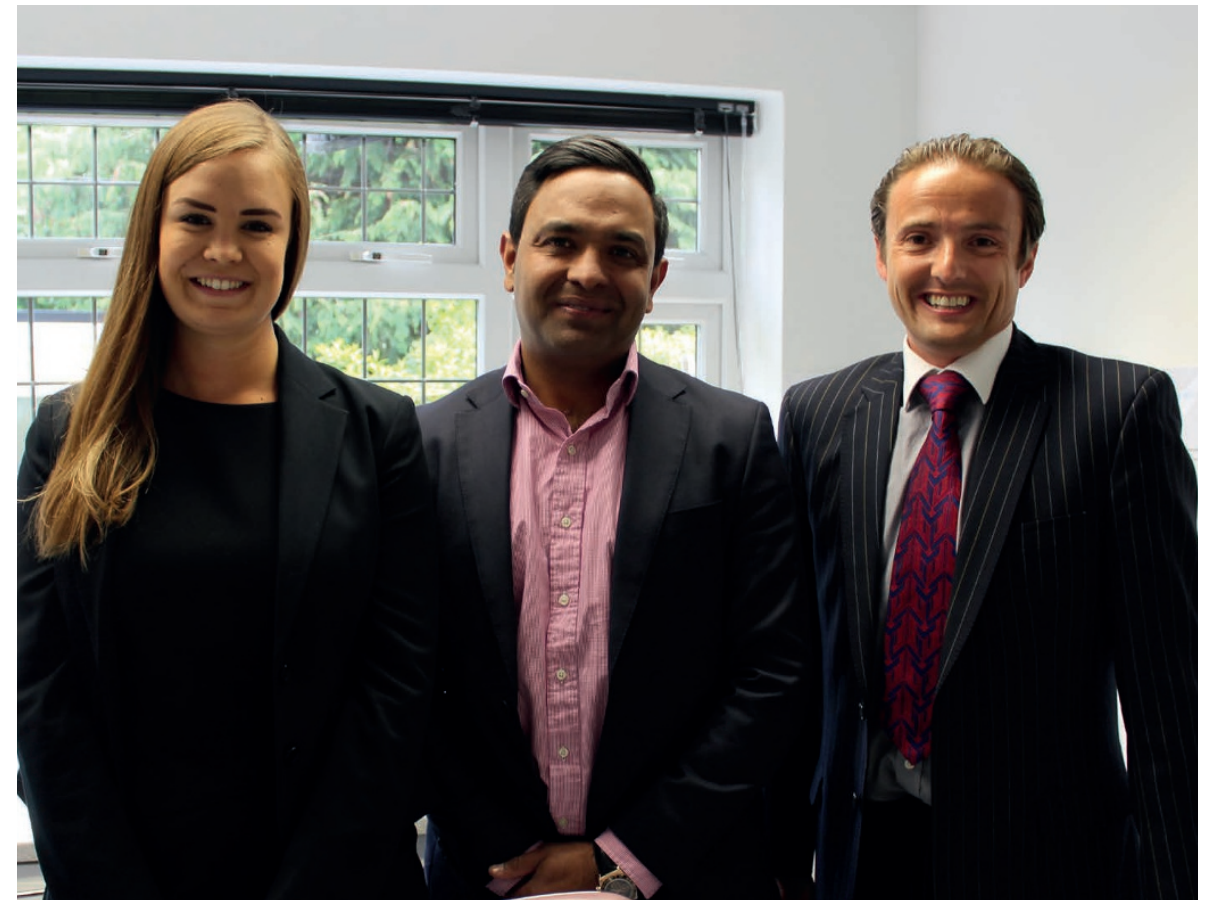

practices around Yorkshire since we began in 2004, and have ambitions to, ultimately, run practices across the North of England. But, it's important to us that each of our practices is run independently to help us give a personal service and to build lasting relationships with our clients. The team at FTA Law continues to provide us with expert legal advice in support of our continued growth plans.'

\section{New microbiome-balancing toothpaste launches in the UK}

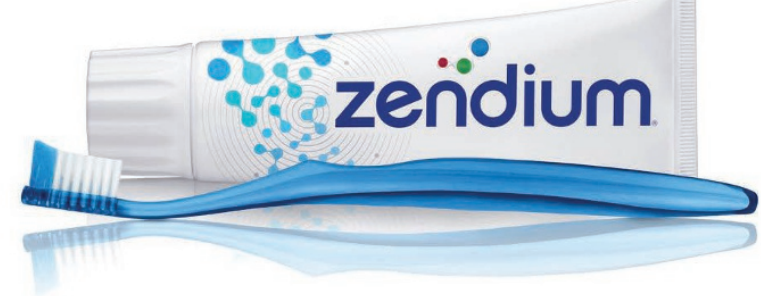

Unilever's premium daily toothpaste, Zendium, will be available in the UK via Amazon, from June 2018. Originally developed over 40 years ago and now available across 18 European countries, Zendium is Scandinavian dentists' most trusted toothpaste brand. ${ }^{1}$

Zendium is a toothpaste that works differently. In addition to fluoride, its unique SLS-free formulation contains natural enzymes and proteins which boost the natural salivary defences ${ }^{2}$ and balance the oral microbiome. A landmark study, published in 2017, showed that over 14 weeks' use, Zendium significantly increased health-associated bacteria and significantly reduced disease-associated bacteria. ${ }^{3}$

These findings complement a growing expert consensus which recognises that it is the overall balance of the oral microbiome which is key to oral health rather than the simple presence or absence of a specific pathogen. ${ }^{4}$

Two further studies now demonstrate the clinical benefit of Zendium's balancing effect. In a 13 -week controlled clinical trial, $83 \%$ of Zendium users experienced improved gingival health with Zendium showing superiority vs control across all three clinical indices measured. ${ }^{5}$ Supporting these results, a real-world study of over 300 people in Denmark revealed that long-term Zendium users had significantly better gingival health than those using regular toothpastes. ${ }^{6}$

1. IPSOS. Dentist survey in Denmark, Sweden and Norway, October 2017

2. Unilever data on file

3. Adams S E, Arnold D, Murphy B et al. A randomised clinical study to determine the effect of a toothpaste containing enzymes and proteins on plaque oral microbiome ecology. Sci Rep 2017; 7: 43344.

4. Kilian M, Chapple I L, Hannig M et al. The oral microbiome - an update for oral healthcare professionals. Br Dent J 2016; 221: 657-666.

5. West N, Newcombe R, Mantzourani M et al. A toothpaste containing enzymes and proteins improves gingival health. In CED-IADR/NOF Oral Health Research Congress September 21-13, 2017: Abstract Book. 0527. International Association for Dental Research, 2017.

6. Pedersen A L, Belstrøm D, Nicholson J et al. Gingival health status in individuals using different types of toothpaste. In CED-IADR/NOF Oral Health Research Congress September 21-23, 2017: Abstract Book. 0472. International Association for Dental Research, 2017. 